\title{
Clinical agreement in pulmonary nontuberculous mycobacterial disease
}

This article was published in the following Dove Press journal:

Clinical Audit

6 November 2013

Number of times this article has been viewed

\section{Peter J Nolan}

Department of Internal Medicine, Toowoomba General Hospital, Toowoomba, Queensland, Australia
Correspondence: Peter J Nolan Department of Internal Medicine, Toowoomba General Hospital, Pechey Street, Toowoomba, Queensland, Australia 4350 Tel +610747380117

$\mathrm{Fax}+610746380917$

Email peter.nolan59@me.com
Purpose: To consider the clinical agreement among respiratory and infectious disease physicians, working in a tertiary chest diseases center serving a population with a low incidence of pulmonary tuberculosis ( $<3 / 100,000$ /year), in the assessment of cases of pulmonary nontuberculous mycobacterial (NTM) lung disease.

Method: A series of previously notified cases of NTM disease was abstracted and anonymously presented to a cohort of seven respiratory and infectious disease physicians. Their individual decisions to notify, treat, and follow the cases was evaluated and compared using the intraclass correlation coefficient.

Results: A wide range was demonstrated in the diagnostic and management decision triage of each case by the physicians participating in the study. Clinical agreement on the likelihood of disease was limited, with an intraclass correlation coefficient of 0.394 . Indication to notify the case to the state registry was linked to the clinical intent to initiate a treatment program.

Conclusion: There appears to be limited agreement on the clinical significance of NTM isolates from pulmonary specimens among this cohort of experienced clinicians. If this trend is generalizable to a wider population of respiratory and infectious disease physicians, the number of notified and treated cases of disease is likely to be an underestimate of the true burden of disease in the general population.

Keywords: diagnostic certainty, Kappa index, intraclass correlation coefficient, lung disease

\section{Introduction}

Nontuberculous mycobacteria (NTM) are ubiquitous pathogens found in soil, tap water, and animals. Exposure infrequently results in clinical disease, which ranges in presentation from necrotizing superficial ulcers and pulmonary suppuration and fibrosis to disseminated disease in the immunosuppressed. ${ }^{1}$ Clinically and radiologically, pulmonary NTM is often indistinguishable from infection by mycobacterium tuberculosis. However, the bacteriology, natural history, and outcome of treatment are significantly different, resulting in different implications for public health authorities. While pulmonary NTM is not infectious, tuberculosis poses a significant risk to the general community and its prompt identification and control is imperative to the maintenance of a low incidence of disease. In contrast, by virtue of its chronicity and poor response to therapeutic intervention, human NTM disease causes significant morbidity within the wider community. Furthermore, over the last 20 years, there has been an increased incidence in Western communities, reflecting an aging population and the impact of Human immunodeficiency virus (HIV) and other forms of immunosuppression. ${ }^{2}$ Major questions about the ecology, transmission, 
pathogenesis, transmission, and treatment of NTM disease will only be addressed if effective monitoring of human disease occurs.

Disagreement over clinical findings, diagnosis, and management decisions are unavoidable in medicine. It is, however, necessary to ascertain whether this variability is significant enough to influence assessment of outcomes, especially when dealing with a notifiable disease. Though definitions exist for the identification of cases of pulmonary NTM disease, the interpretation of these criteria varies and this may ultimately influence the notification rate of disease to public health authorities and the clinical decision to consider treatment. ${ }^{3}$

Traditionally, assessment of clinical agreement has been determined through the calculation of the Kappa $(\mathrm{K})$ coefficient. Kappa values range from -1 to +1 with a value of +1 indicating near perfect agreement between raters and a value of zero being interpreted as chance agreement. ${ }^{4,5}$ Values less than zero suggest worse than chance agreement. ${ }^{6,9}$

This study outlines a revised process of accessing clinical agreement and focuses on the clinical evaluation of NTM by a cohort of respiratory and infectious disease physicians.

\section{Methods}

Ten notified cases of NTM were chosen at random from records available through the Statewide Chest Service. Cases were only included if:

1. Microbiology and clinical record including management were complete ( six cases of Mycobacterium aviumintracellulare, two cases of Mycobacterium kansasii, one case of Mycobacterium fortuitum, and one case of Mycobacterium simiae).

2. Original presenting chest X-ray was available.

3. Informed consent was obtained.

Seven thoracic and infectious disease physicians experienced in the management of pulmonary NTM disease were independently asked to review each deidentified case record with the accompanying X-ray and to complete a simple questionnaire on three aspects of the case management. These three aspects, believed to be critical to the accuracy of disease notification included:

1. Their opinion as to the likelihood of significant disease.

2. Whether they would notify the case.

3. What initial treatment would be instituted.

All candidates were blind to the actual outcome of each case. The researcher was present to clarify any details in the case histories. Copied X-rays were of excellent quality. The study was approved by the Prince Charles
Hospital Regional Health Service Research and Ethics Committee.

\section{Analysis}

Results were tabulated on SPSS (Version 16) for Windows (IBM Corporation, Armonk, NY, USA) following coding of each case. Physicians were compared in their assessment of each case and in their overall assessment of the ten cases using parametric analysis of mean and standard deviation, looking for systematic patterns of disagreement between physicians. Clinical agreement was assessed with a factor analysis of variance followed by calculation of the intraclass correlation coefficient (ICC) using the method described by Bartko. $^{7}$

Fleiss has indicated that this version of the ICC is equivalent to Kappa provided that the number of subjects is not very small and that an appropriately weighted form of Kappa is used to take account of the ordinal nature of the data. ${ }^{8}$ Weighting was felt to be equivalent in this case as each decision led to an independent outcome. Significance of Kappa (ICC) was calculated from the $F$-statistic where $F=$ mean sum of squares/mean square error with $F$ distribution $(n-1) \times(m-1)$. The assumptions for the use of Kappa (ICC) were fulfilled including:

1. Physician and cases were independent of each other.

2. Physicians operated independently.

3. The evaluative categories are mutually exclusive.

\section{Results}

Each decision was coded and cross tabulated for further analysis. Data was complete for all cases and for all decision options by each physician. There was a wide variation in the clinical estimate of disease likelihood scored by each physician for each case (Table 1). It is to be noted that each of the ten cases had been notified to the Notifiable Disease Registry in the Statewide Chest Clinic and indeed some of the participating physicians had been the notifier of four of the cases. The physicians were blinded to this possibility and showed no evidence of recognition of any case. Though there was relatively close agreement on the likelihood of clinically significant disease in case eight, the remainder of the cases lacked this uniformity. Assessment of clinical agreement for all cases by the seven physicians was undertaken using a two way factor analysis of variance and estimate of the ICC as an estimate of Kappa (Table 2). The estimated ICC was 0.394 , indicating limited agreement between the seven physicians as to the likelihood of clinically significant NTM pulmonary disease in the ten cases. It is possible that this 
Table I Distribution of responses by the seven physicians when determining the likelihood of pulmonary NTM disease for the ten cases surveyed

\begin{tabular}{lllll}
\hline Case & Observations & Mean & SD & Range \\
\hline 1 & 7 & 2.7 & 1.25 & $\mathrm{I}-4$ \\
2 & 7 & 3.6 & 0.79 & $3-5$ \\
3 & 7 & 2.3 & 0.95 & $1-4$ \\
4 & 7 & 3.3 & 0.76 & $2-4$ \\
5 & 7 & 2.7 & 0.95 & $1-4$ \\
6 & 7 & 3.0 & 1.0 & $2-4$ \\
7 & 7 & 2.0 & 0.82 & $1-3$ \\
8 & 7 & 4.3 & 0.49 & $4-5$ \\
9 & 7 & 4.1 & 0.69 & $3-5$ \\
10 & 7 & 2.6 & 0.79 & $2-4$ \\
\hline
\end{tabular}

Abbreviations: NTM, nontuberculous mycobacterial; SD, standard deviation.

estimate of agreement would have been influenced by a weighting system that either excluded or gave less weight to the option of an indeterminate estimate of disease (ie, those cases requiring further evaluation). Only in one of 21 instances of indeterminate diagnostic certainty of disease did the physician nominate that they would notify the case to the Notifiable Disease Registry. There appeared to be a relationship between the estimate of clinical likelihood of disease and the intention to notify the case to the disease registry (Table 3). Physicians who evaluated a case as indicating likely or definite pulmonary disease in association with the NTM isolate were more likely to notify the case compared to those physicians who evaluated the cases as being unlikely to relate to significant pulmonary disease or suggested that the case required further evaluation. Management was similarly related to the intention to notify the case. Cases that physicians nominated as requiring treatment were more likely to be notified compared to those cases where management involved only long-term or short-term observation by a specialist or general practitioners (Table 4). The relationship between the recommended management plan and the individual physician's estimate of the clinical likelihood of significant disease was quite diverse. Even in cases where the physician felt that disease was likely to exist $(n=22)$, only eleven were recommended to receive definitive

Table 2 Two-way factor analysis of variance (balanced design without replication)

\begin{tabular}{llllll}
\hline $\begin{array}{l}\text { Sources of } \\
\text { variation }\end{array}$ & $\begin{array}{l}\text { Degrees of } \\
\text { freedom }\end{array}$ & $\begin{array}{l}\text { Sum of } \\
\text { squares }\end{array}$ & $\begin{array}{l}\text { Mean } \\
\text { squares }\end{array}$ & $\boldsymbol{F}$ & \\
\hline Physician & 6 & 6.171 & 1.029 & 1.415 & $P>0.05$ \\
Cases & 9 & 36.343 & 4.038 & 5.55 & $P<0.05$ \\
Interaction & 54 & 39.257 & 0.727 & - & 0.001 \\
Total & 69 & 81.771 & & & \\
\hline
\end{tabular}

Table 3 Relationship between estimate of clinical likelihood of disease and notification intent by seven physicians assessing ten cases of pulmonary nontuberculous mycobacterial disease

\begin{tabular}{llll}
\hline Clinical estimate of likelihood & \multicolumn{3}{l}{ Notification intent } \\
\cline { 2 - 4 } of disease & Yes & Unsure & No \\
\hline Definite disease & 5 & - & - \\
Likely disease & 21 & - & 1 \\
Requires further evaluation & $\mathrm{I}$ & 5 & 15 \\
Unlikely disease & $\mathrm{I}$ & - & 15 \\
No disease & - & - & 6 \\
\hline
\end{tabular}

therapy (chemotherapy and/or surgery). The most common recommended management plan was specialist observation. It is possible that these trends reflect the commonly held opinion by clinicians that the disease is difficult to eradicate, that the therapy is toxic, and that the clinical benefit for the patient is often small.

\section{Discussion}

Human NTM disease does not fit neatly into the standard guidelines for a notifiable disease. Firstly, person to person transmission does not occur, the exact environmental exposure that leads to infection is unknown and may vary from patient to patient, and finally, effective management strategies are not agreed upon. The disease manifestations are quite diverse with new variants emerging over the last 20 years largely as a result of the global epidemic of HIV disease. In the state of Queensland, NTM disease is notifiable and since 1988, laboratory based notification has been mandatory under state legislation.

The problem of clinical agreement in pulmonary NTM disease is paramount in determining the effectiveness of current surveillance techniques to accurately determine the true incidence of this disease in the community. It can be seen that there is a close relationship between an individual physician's clinical assessment of the likelihood of disease and their intention to notify the case to the Notifiable Disease Registry. It is possible therefore, that true cases may not be

Table 4 Relationship between management plan and notification intent by seven physicians assessing ten cases of pulmonary nontuberculous mycobacterial disease

\begin{tabular}{llll}
\hline Management plan & \multicolumn{3}{l}{ Notification intent } \\
\cline { 2 - 4 } & Yes & Unsure & No \\
\hline General physician observation & I & - & 8 \\
Specialist observations & 12 & 5 & 27 \\
Chemotherapy & I5 & - & - \\
Surgery & I & - & - \\
No follow-up & - & - & I \\
\hline
\end{tabular}


referred because initial evaluation resulted in uncertainty as to the clinical likelihood of disease.

The estimate of the clinical likelihood of disease appears to vary considerably from physician to physician in the ten cases that were surveyed. The ICC was chosen to enable approximation of Kappa because of its ease in dealing with ordered categories (or choices of diagnosis and treatment) when there are two or more judges. The estimated result of agreement among physicians (ICC $=0.394$ ) only indicates a "fair" agreement among physicians. This estimate is disturbingly low when compared to a Kappa of 0.42 for observer estimate of jugular venous pressure, 0.50 for observer agreement in the presence or absence of a dorsalis pedis pulse, and 0.62 for the presence or absence of various signs of chronic airflow obstruction. ${ }^{6,9}$

This study is limited in its internal validity by the small number of patients and observers, the problem of selection bias in the cases chosen by the author and the presence of the author at the time of the evaluation by each of the observers. A larger selection of cases and a wider survey of the community of practicing respiratory and infectious disease physicians is required to confirm these preliminary findings. If such diversity of opinion is consistently found, then there may be a case for re-evaluation of clinical diagnostic criteria for pulmonary NTM disease and education of the referring medical community in order to improve the evaluation of patients with isolates of NTM from respiratory secretions.

The actual community prevalence and morbidity from pulmonary NTM disease is likely to be underestimated on the basis of this survey of a cohort of respiratory and infectious disease physicians. If more suitable and effective chemotherapeutic regimens are to be developed and evaluated, there needs to be greater consensus among clinicians about whether an NTM isolate represents disease or asymptomatic colonization.

\section{Acknowledgments}

The assistance and support of colleagues in the Department of Thoracic Medicine at the Prince Charles Hospital, Chermside, and The Brisbane Chest Clinic, Division of Specialised Services, Queensland Health is gratefully acknowledged.

\section{Disclosure}

The author reports no conflicts of interest in this work.

\section{References}

1. Iseman MD, Marras TK. The importance of nontuberculous mycobacterial lung disease. Am J Respir Crit Care Med. 2008;178(10): 999-1000.

2. Horsburgh CR Jr, Selik RM. The epidemiology of disseminated nontuberculous mycobacterial infection in the acquired immunodeficiency syndrome (AIDS). Am Rev Respir Dis. 1989;139(1):4-7.

3. Griffith DE, Aksamit T, Brown-Elliott BA, et al; ATS Mycobacterial Diseases Subcommittee; American Thoracic Society; Infectious Disease Society of America. An official ATS/IDSA statement: diagnosis, treatment, and prevention of nontuberculous mycobacterial diseases. Am J Respir Crit Care Med. 2007;175(4):367-416.

4. Jeyaseelan L, Rao PS. Statistical measures of clinical agreement. Natl Med J India. 1992;5(6):286-290.

5. Cyr L, Francis K. Measures of clinical agreement for nominal and categorical data: the kappa coefficient. Comput Biol Med. 1992;22(4): 239-246.

6. Landis JR, Koch GG. The measurement of observer agreement for categorical data. Biometrics. 1977;33(1):159-174.

7. Bartko JJ. The intraclass correlation coefficient as a measure of reliability. Psychol Rep. 1966;19(1):3-11.

8. The measurement of Interrater Agreement. In: Fleiss JL, Levin B, Paik MC, editors. Statisitcal Methods for Rates and Proportions, 3rd ed. New York: John Wiley \& Sons Inc. 2004;598-626.

9. Sackett DL, Haynes RB, Guyatt GH, Tugwell P. Clinical Epidemiology: A Basic Science for Clinical Medicine. 2nd ed. New York: Little, Brown and Company; 1991.
Clinical Audit

\section{Publish your work in this journal}

Clinical Audit is an international, peer-reviewed, open access journal focusing on the processes and outcomes of clinical audit in any area of healthcare. All aspects of patient care are addressed within the journal and practitioners from all disciplines are invited to submit their work. Areas covered include: Publication of audits; How an audit has changed practice; Submit your manuscript here: http://www.dovepress.com/clinical-audit-journal

\section{Dovepress}

Practical tips on how to do audits and to avoid pitfalls; How audits have changed patient care; Calls and justifications for new audits. The manuscript management system is completely online and includes a very quick and fair peer-review system, which is all easy to use. Visit http://www.dovepress. com/testimonials.php to read real quotes from published authors. 